Bulgarian Academy of Sciences. Space Research and Technology Institute.

Aerospace Research in Bulgaria. 32, 2020, Sofia

DOI: https://doi.org/10.3897/arb.v32.e11

\title{
2.5D LES SIMULATION OF AN AIRFOIL SHOCK WAVE REDUCTION BY USING POROUS MEDIA
}

\author{
Ion Malael, Ioana Octavia Bucur, Valeriu Dragan \\ National Research and Development Institute for Gas Turbines COMOTI, Romania \\ e-mail:ioana.bucur@comoti.ro
}

Key words: Airfoil, Numeric Simulations

\begin{abstract}
Supersonic flight has become a practical reality since the 1950s. One of the first ways to study high speed effects of the shock waves is to evaluate the aerodynamic coefficients of an airfoil. The work described herein refers to a series of 2.5D LES numeric simulations, to investigate the behavior of the shock wave on the airfoil. To reduce the unwanted effects, a porous surface is placed on $80 \%$ of suction and pressure side of a NACA 0012 airfoil. Solving the motion equations was carried out with Ansys Fluent. Qualitative comparison consists in the pressure contours visualization for different angles of attack, showing how shock waves form on the airfoil surfaces. After plotting the polar diagrams, $C L=f(A o A)$ and $C L=f(C D)$, a quantitative comparison was made between the baseline airfoil and the same airfoil but with porous media on each surface side.
\end{abstract}

\section{Introduction}

Current literature regarding porous media focuses mainly on the flow through such a medium and its engineering applications, such as thermal transfer or acoustic lining [1]. One approach to define and characterize porous media is presented by W. Ehlers and J. Bluhm [2]. A review of thin porous media state of the art is briefly analyzed in [3], with a main focus on new developed theories that address the matter, such as GDL (Gas diffusion layers), MPN (molecular pore network), PTM (pore topology method). With this baseline, experiments and numerical applications were conducted in the medical field and biotechnology $[4,5]$ and innovative technologies in the energetic field [6]. Recent studies show how applications of porous media can improve flow in a wind tunnel. Khalid et al. use porous boundaries and manage to obtain better results when simulating the flow through a wind tunnel. Porous media was used in their paper [7] to reduce the wall interference effects.

A lesser known application of porous media is the behavior of shock waves in regard to it and may provide genuine optimization solutions. A study referring to steady shock waves in porous aluminum that concentrates mainly on the interaction 
between the metal and the shock wave is presented in [8]. Its conclusions sustain the theory that porous metals help in the mitigation of shock waves formation. Furthermore, alongside with researches regarding porous metals, there are also papers analyzing the behavior of shock waves in porous plastic solids [9]. Cohen and Durban attempt to determine the influence of porosity on plastic solids using as a baseline the Gurson model from 1977. Their findings in [9] conclude that porous medium can influence the presence of shock waves by delaying their appearance and also such a medium slows down their propagation speed.

G. Savu published his research regarding the porous airfoil in transonic flow in the early 80 's. He and his colleague carried on numerical and experimental work on the behavior of a porous airfoil with a plenum chamber placed on the suction side. They observed, in a supersonic wind tunnel, that the shock wave formed on the upper surface of the airfoil can be splitted in smaller waves by using porous media on that surface [10].

Another relevant point of view is offered by Gubin in his paper [11], in which he states that when the porous area expands and becomes greater than that of the shock wave, the technique will have a greater impact.

In order to study the flow over an airfoil with porous media on the suction side it is necessary to review the patterns regarding shock wave formation over a standard airfoil model. This phenomenon is analyzed in [12], where the author uses a NACA 0012 airfoil and considers angles of attack varying from $0^{\circ}$ to $5^{\circ}$ in a transonic regime with Mach numbers in the range of 0.2 and 0.8 .

In previous years, due to software development in the field of computational fluid dynamics, the technology advances made it possible to detect shock waves through some specific methods, more accurately than in the early case studies. These methods include density gradient maxima, normal Mach number and characteristics which are briefly presented with their advantages and disadvantages in [13]. Based on these methods, researchers have determined other optimized ways to detect shock waves, taking into account its mathematical definition and implying the use of eigenvectors and the Riemann invariants [14].

The 2.5D LES (Large Eddy Simulations) are known to give more accurate results and be more efficient in comparison with other CFD approaches, such as 2D URANS (Unsteady Reynolds-Averaged Navier-Stokes) or 2.5D URANS, in terms of aerodynamic performance evaluations [15-17].

This paper is aiming to investigate the behavior of the shock wave formed on an airfoil placed in a high speed flow. The 2.5D LES simulations was carried to cover a set of geometrical and aerodynamic configurations representative for shock wave regime.

\section{CFD Setup}

With a physics-based understanding of the flow instabilities that occur at high speeds over an airfoil, we can develop a viable control technology for flow 
instabilities that will allow the airfoil to operate at an increased efficiency in that regime. Due to the dimensional nature of the unsteady aerodynamic phenomenon occurring during the appearance of a shock wave, the full extent of the implications and interactions can be captured by fully viscous $2.5 / 3 \mathrm{D}$ unsteady CFD simulations. However, lower order models are known to provide valuable guidelines regarding the properties of unstable flow patterns in flow around airfoils.

High-fidelity computational approaches like Large Eddy Simulation (LES) [18] or Detached Eddy Simulation (DES) [19] are suitable for understanding flow dynamics associated with aerodynamic flows. They are resolving (not modelling) a large range of flow scales.

The CFD used in this study integrates the LES discretized fully compressible Navier-Stokes equations describing the conservation of mass, momentum and total energy. Therefore, the Navier-Stokes equations have to be filtered with respect to the grid size in order to obtain the LES governing equations [20].

$$
\begin{gathered}
\frac{\partial \bar{u}_{i}}{\partial x_{i}}=0 \\
\frac{\partial \bar{u}_{i}}{\partial t}+\frac{\partial}{\partial x_{j}}\left(\overline{u_{\imath} u_{j}}\right)= \\
=-\frac{1}{\rho} \frac{\partial \bar{p}}{\partial x_{i}}+v \frac{\partial}{\partial x_{j}}\left(\frac{\partial \bar{u}_{i}}{\partial x_{j}}+\frac{\partial \bar{u}_{j}}{\partial x_{i}}\right)= \\
=-\frac{1}{\rho} \frac{\partial \bar{p}}{\partial x_{i}}+2 v \frac{\partial}{\partial x_{j}} S_{i j}
\end{gathered}
$$

In this paper three cases were numerically investigated starting with the NACA 0012 baseline airfoil. In the other two cases the structure of porous media was changed: in the first case the airfoil was perforated on $80 \%$ of its upper/lower surface, fourteen times and in the second case the airfoil has twenty-six holes to simulate a porous media. Fig. 1 presents the geometries of the studied cases.

a)

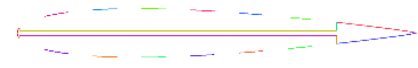

b) c)

Fig. 1. Cases geometry: a) baseline, b) porous media case with 14 holes, c) porous media case with 26 holes

Accurate CFD methods require domains large enough to minimize the boundary effects on the resulted prediction of the shock wave evolution. In Fig. 2, the computational domain used in all three simulations is presented. In terms of boundary conditions, pressure far field condition with Mach number value at the domain border was imposed while for the airfoil, a no-slip wall BC was used. 


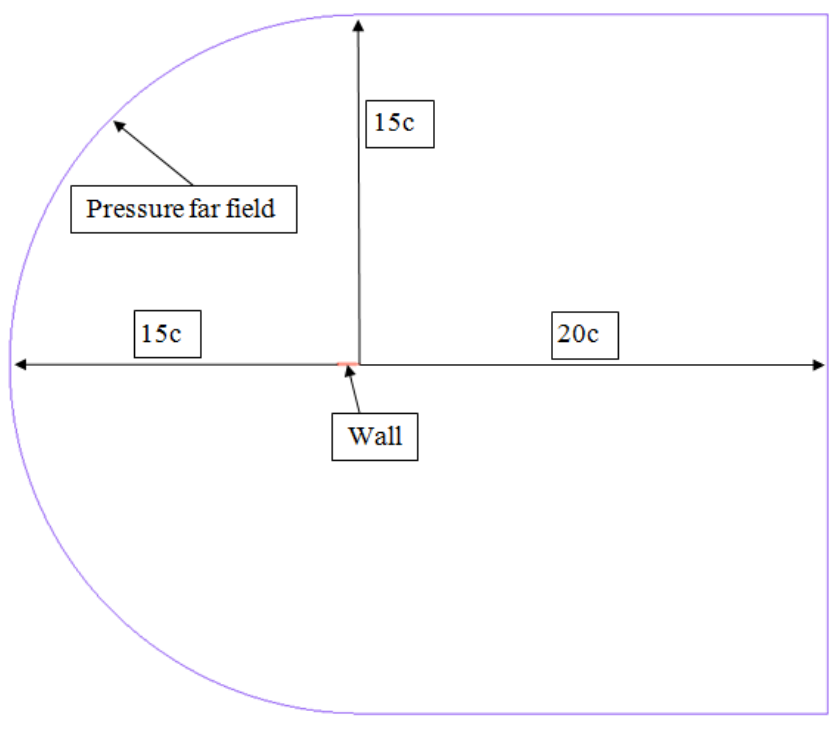

Fig. 2. Computational domain

Mesh quality is a major factor in order to avoid slow convergence, or even convergence problems. Because of its complex dimensional structure, producing a good mesh of is not trivial.

The mesh was generated with ICEM CFD using block-structured meshing, controlled in terms of skewness, growth and aspect ratio. For representation purposes only, a course mesh generated by ICEM CFD for this study can be found hereafter.

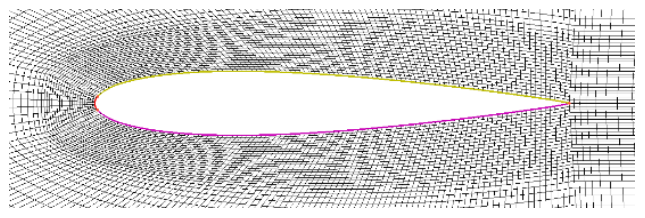

a)

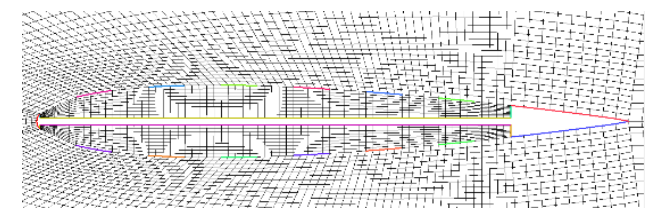

b)

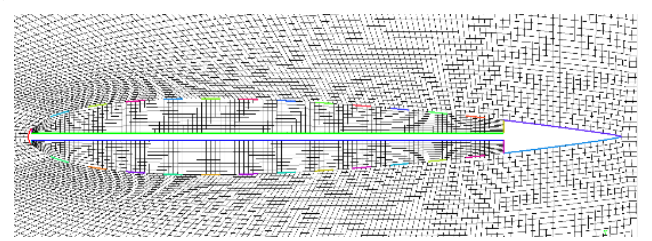

c)

Fig. 3. Mesh: a) baseline, b) 14 holes porous media, c) 26 holes porous media. 
The target final mesh size will be about 2.5 million grid cells for a case, with a ratio between two neighbour cells around 1.05. The first wall cell size was defined in order to reach a $\mathrm{y}^{+}$of the order of unity at the first point away from the solid walls.

\section{Results}

In order to determine the best size and ratio of the porous wall placed on an airfoil, in reducing the shock wave, several simulations were carried out, using the LES methods. It is a well known issue that on the airfoil in a high speed flow the shock wave has a bad influence on aerodynamic efficiency, generating entropy and leading to boundary layer interactions which are difficult to control in flight. For the baseline, the NACA 0012 was used and after that it was modified for the other two cases, where PM was installed on $80 \%$ of the airfoil surface. In Fig. 4 the static pressure flow field for the three studied cases it's presented at different angle of attack.

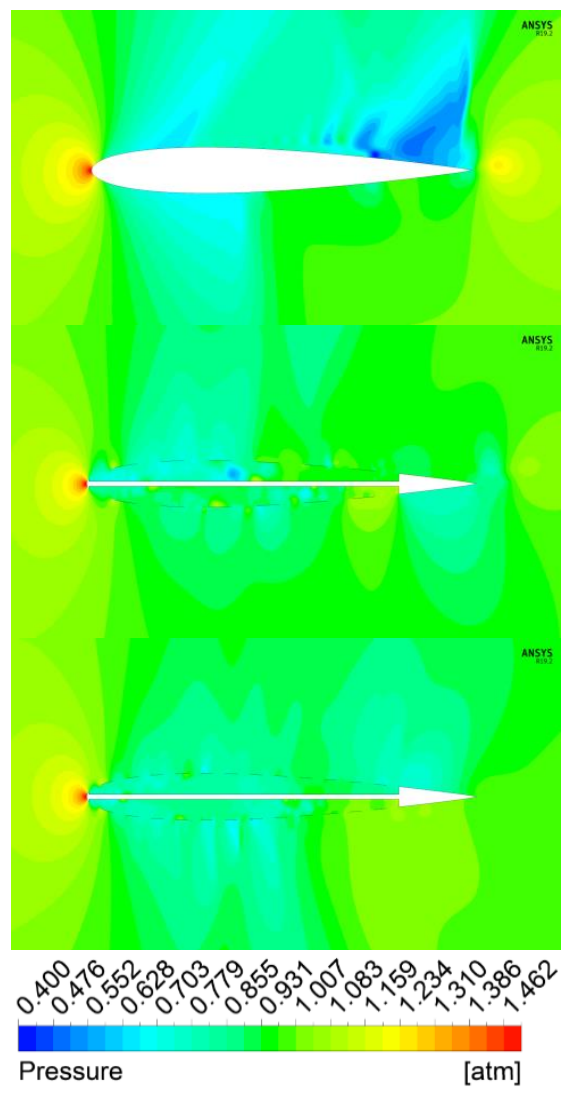

a) $0^{\circ} \mathrm{AoA}$

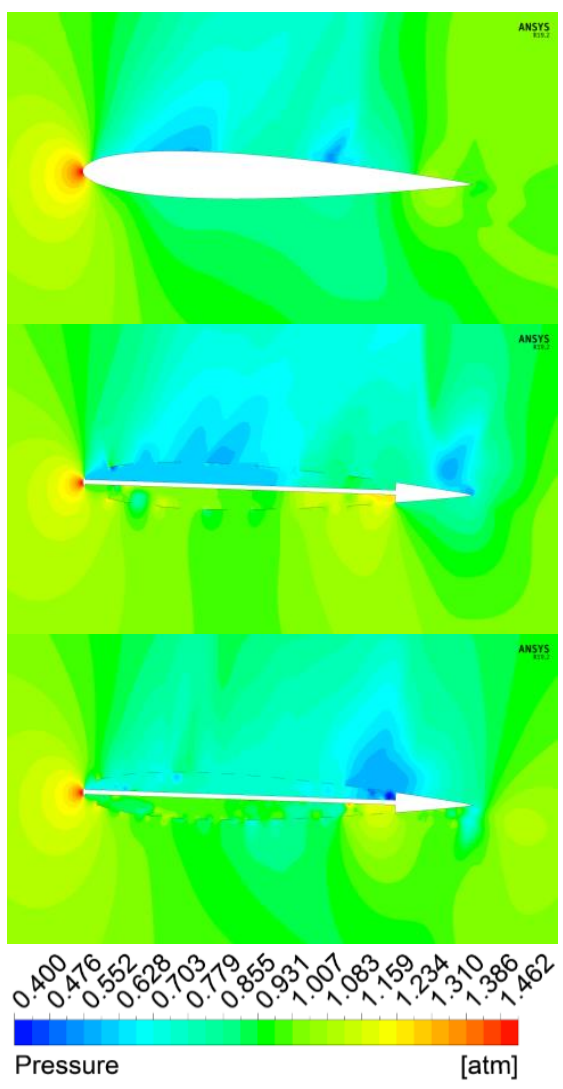

b) $2^{\circ} \mathrm{AoA}$ 


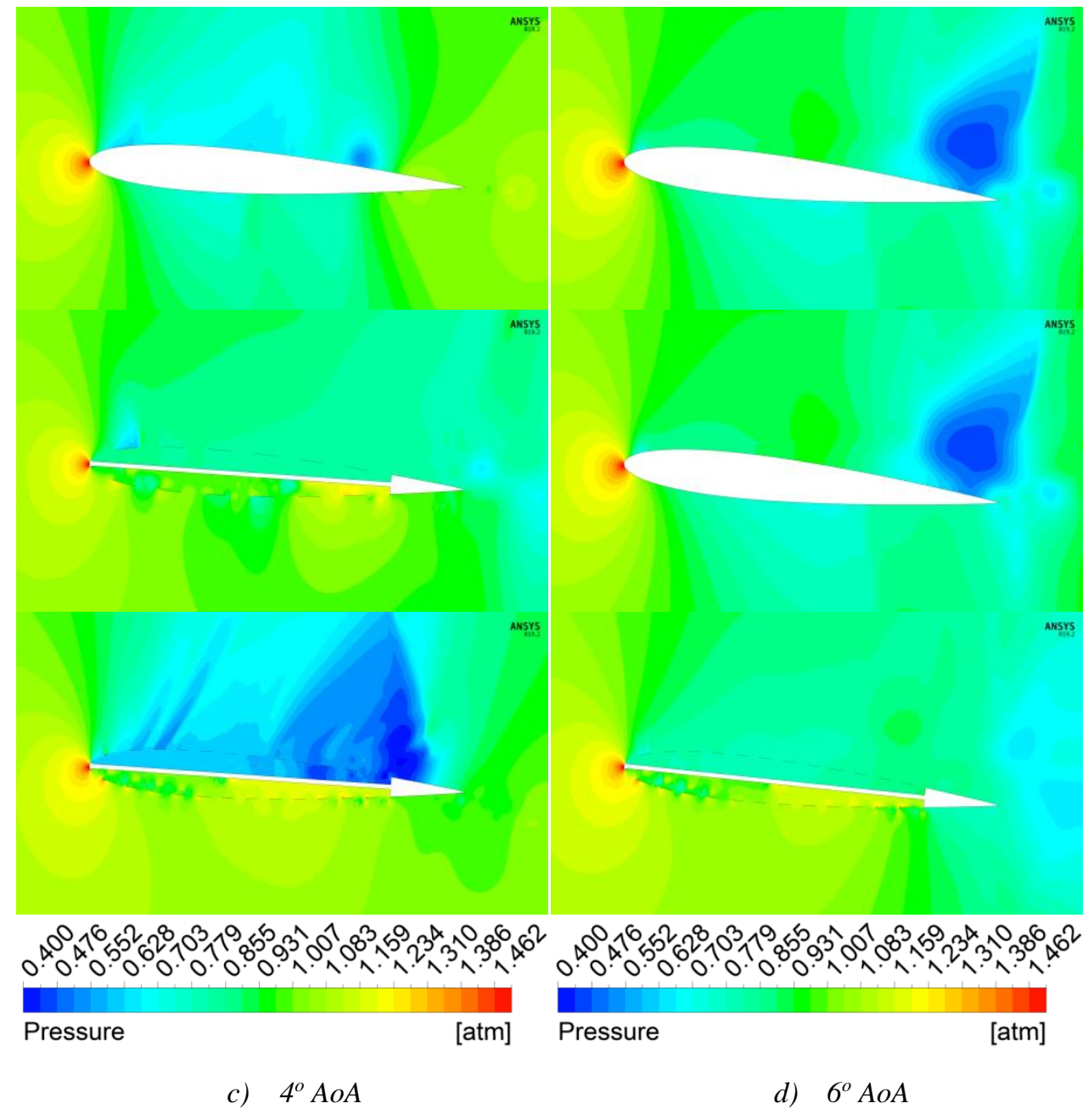




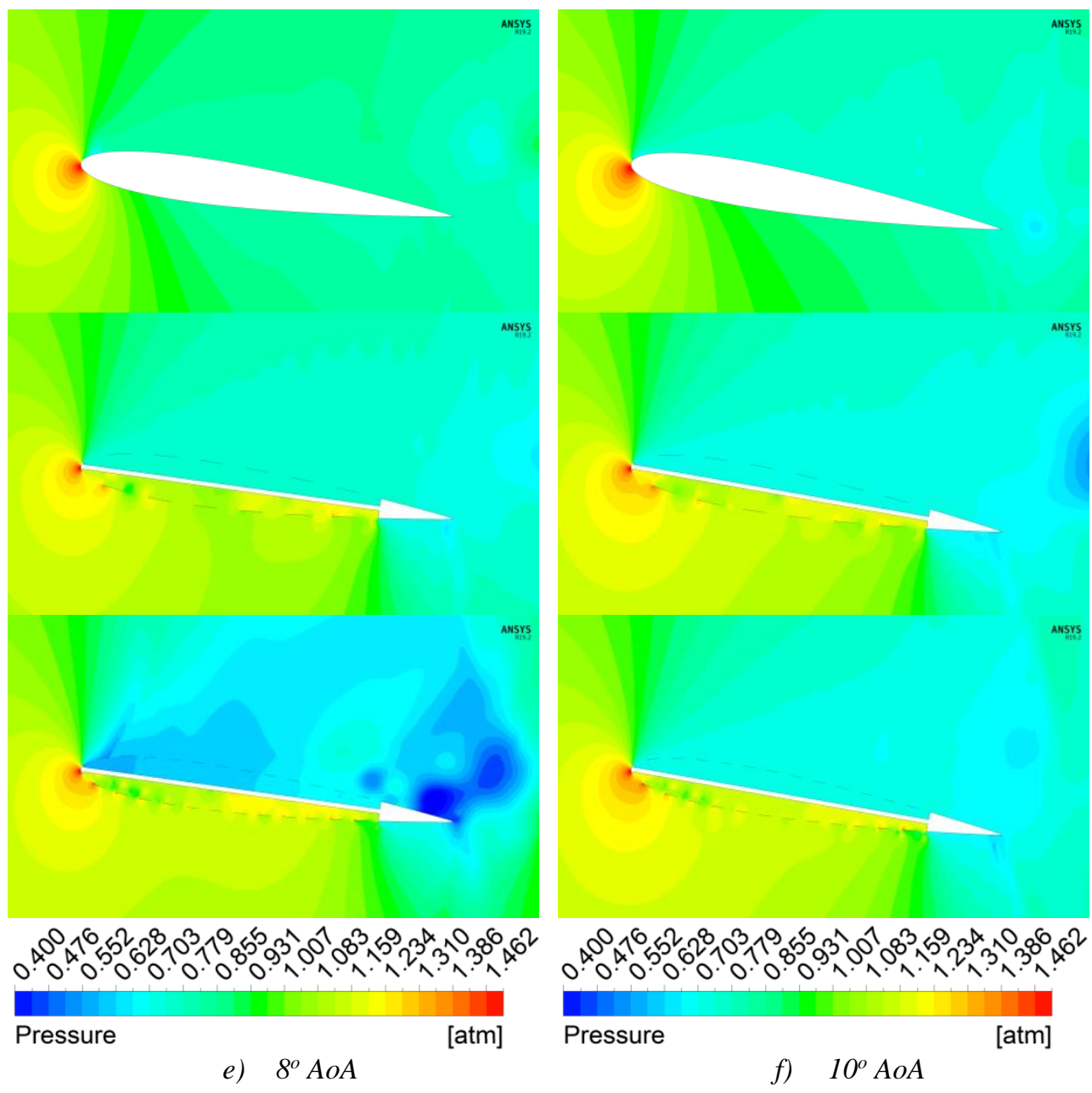




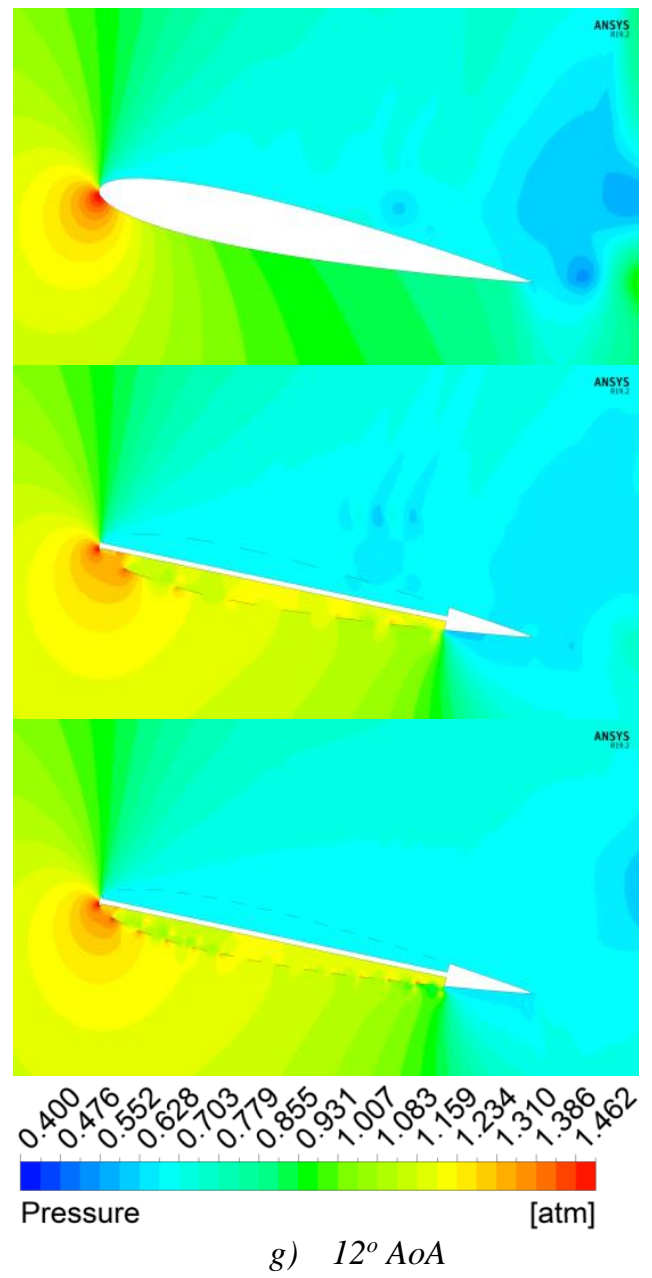

Fig. 4. Static pressure flow field at: a) $\left.0^{\circ} \mathrm{AoA}, \mathrm{b}\right) 2^{\circ} \mathrm{AoA}$, c) $4^{\circ} \mathrm{AoA}$, d) $\left.\left.\left.6^{\circ} \mathrm{AoA}, \mathrm{e}\right) 8^{\circ} \mathrm{AoA}, \mathrm{f}\right) 10^{\circ} \mathrm{AoA}, \mathrm{g}\right) 12^{\circ} \mathrm{AoA}$.

Fig. 5 shows the variation of the lift coefficient for different angles of attack. The maximum value for the airfoils with $\mathrm{PM}$ is obtain at $\mathrm{AOA}=4$ degrees, compared to the baseline where the $\mathrm{CL} \max$ is at $\mathrm{AOA}=2$ degrees. A note must be made that the NACA 4 series is not suitable for high Mach number; hence the atypically low angles of attack. 


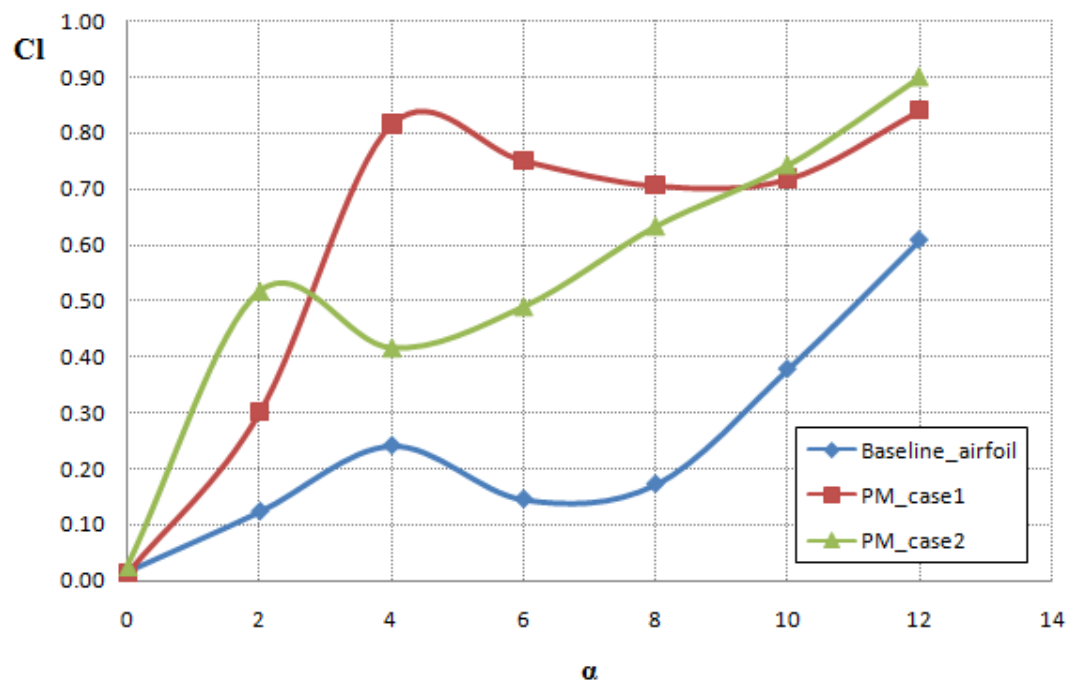

Fig. 5. Lift coefficient variation as function of AoA

Regarding the drag coefficient variation, figures 6 and 7 depict the impact on lift and lift to drag ratio evolution of the various porous configurations.

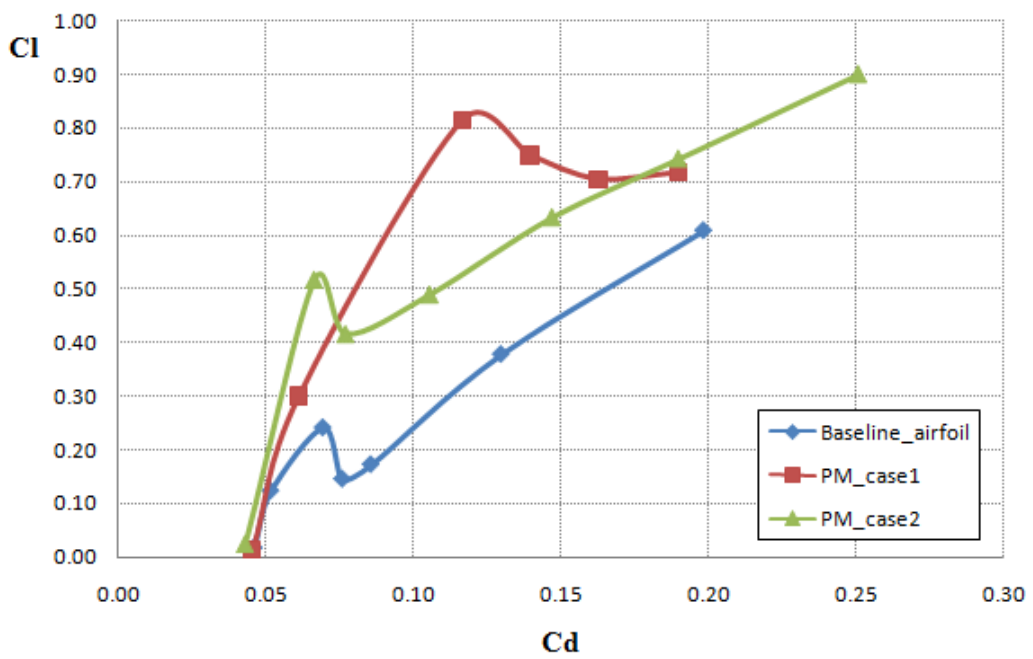

Fig. 6. Lift coefficient variation as function of drag coefficient - note the "drag bucket" extension of $P M 1$ 


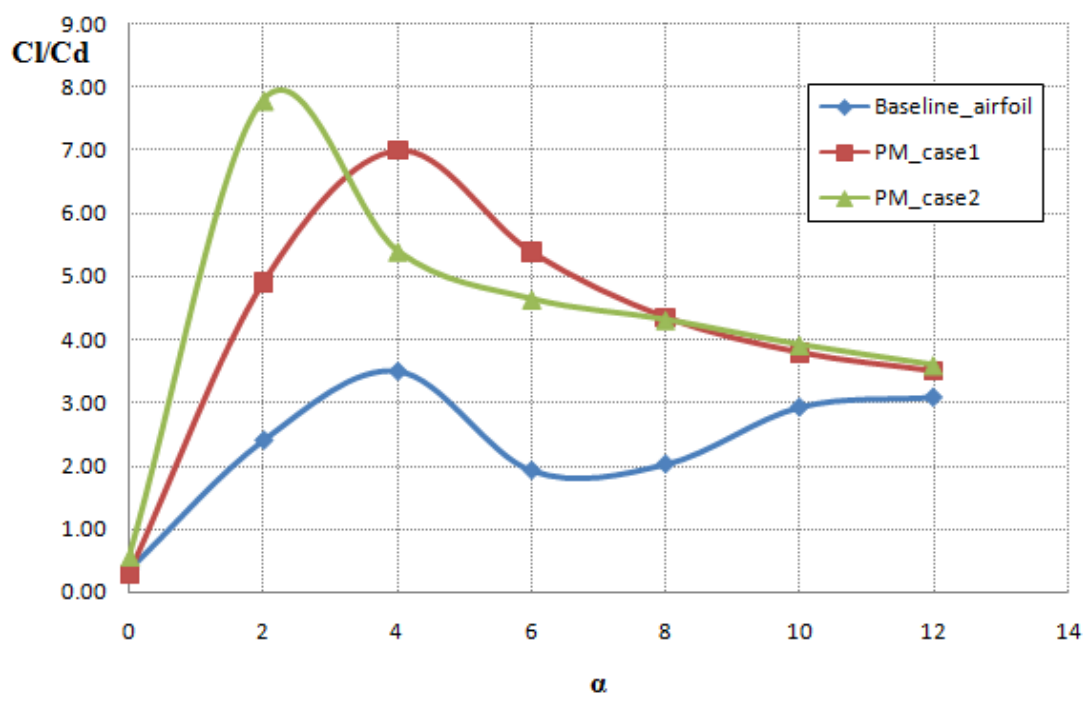

Fig. 7. Lift to Drag ratio variation as function of drag AoA

\section{Conclusion}

The first major conclusion that can be drawn from this study is that porous walls have a definite effect on the high speed aerodynamics of airfoils.

Lift coefficients improved on both cases, with a finer orifice airfoil having a maximum at lower AoA compared to the other cases. Due to the greater size of the orifices, the first porous wall case PM1 behaved similar to a flat plate with a qualitative evolution similar to the baseline - although with better quantitative values. The more fine porous wall, PM2, also showed clear improvement in both lift and lift-to-drag ratio, but with the added note that the peak performance was registered at a lower value of the AoA than the baseline and PM1.

Not very intuitively, the drag bucket of PM2 was more similar to that of the baseline - to the extent that one can define such a notion when using this family of airfoils under these conditions. The coarser orifice wall of PM1 leads to the overall extension of this region of interest, therefore making it more useful to the designers who must factor in stability and range of motion.

It is unclear wheather or not the shape of the plenum is relevant to the overall performance but this parameter is worth further investigation.

\section{Future Work}

As future studies an experimental model of a centrifugal stator with porous media on the blades will be manufactured using the National Research and Development Institute for Gas Turbine - COMOTI technological research 
infrastructure (Fig. 8a). After that a tests campaign will be carried out to validate the numerical results (Fig. 8b).

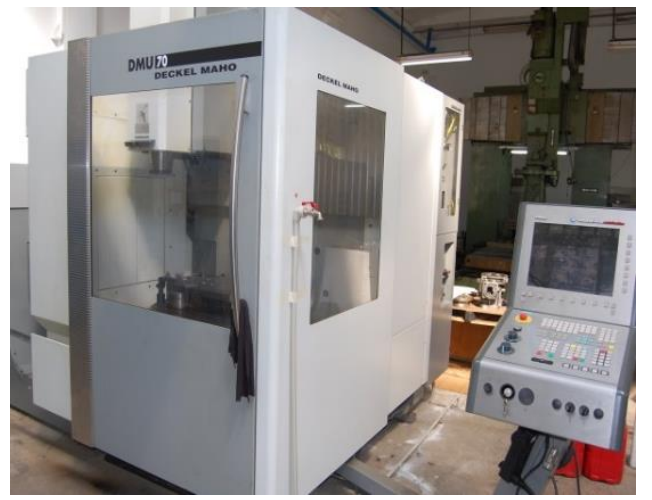

a)

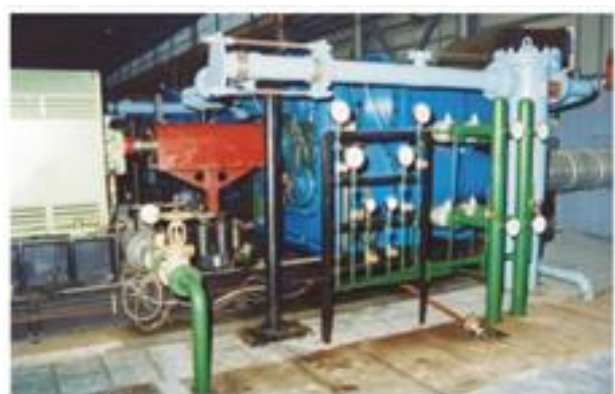

b)

Fig. 8. a) CNDMU 70 Deckel Maho machine, b) NRDI COMOTI centrifugal compressor testing facilities

\section{Acknowledgement}

This work was carried out within "Increasing Excellence in Research Development of RRDI for Gas turbine - COMOTI" Project CREaROR", supported by the Romanian Minister of Research and Innovation, project number Ctr. 3PFE/2018.

\section{References}

1. Bear, J. Dynamics of Fluids in Porous Media. Michigan, American Elsevier Publishing Company, 1972, $764 \mathrm{p}$.

2. Ehlers, W., J. Bluhm. Porous Media: Theory, Experiments and Numerical Applications. Berlin, Springer-Verlag, 2002, 459 p.

3. Yeghiazarian, L., K. Pillai, and R. Rosati. Thin Porous Media. Transport in Porous Media, 2016, 115, 3, 407-10.

4. Vafai, K. Porous Media: Applications in Biological Systems and Biotechnology. Florida, CRC Press, 2010, 632 p.

5. De Lemons, M. Turbulence in Porous Media: Modeling and Applications, Second Edition. Amsterdam, Elsevier, 2012, 408 p.

6. Mujeebu, M. A., M. Z. Abdullah, M. Z. Abu Bakar, A. A. Mohamad, and M. K. Abdullah. Applications of porous media combustion technology - A review. Applied Energy, 2009, 86, 9, 1365-75.

7. Khalid, M., K. A. Juhany, and S. Hafez. Computational modeling of the flow in a wind tunnel. Aircraft Engineering and Aerospace Technology, 2018, 90, 1, 175-85.

8. Czarnota, C., A. Molinari, and S. Mercier. The structure of steady shock waves in porous media. Journal of the Mechanics and Physics of Solids, 2017, 107, 204-28. 
9. Cohen, T., D. Durban. Steady shock waves in porous plastic solids. International Journal of Solids and Structures, 2015, 71, 70-78.

10. Savu, G., O. Trifu. Porous Airfoils in Transonic Flow. American Instritute of Aeronautics and Astronautics Journal, 1984, 22, 7, 989-91.

11. Gubin, S. A. Shock waves in porous media. Jorunal of Physics: Conference Series, 2018, 1099.

12. Leite, H. F., A. C. Avelar, J. B. P. F. Filho, and R. G. A. da Silva. Experimental Study of Shock Wave Patterns over an Airfoil. American Institute of Aeronautics and Astronautics: Conference Paper - Shock Dominated Flows I, 2016, DOI: https://doi.org/10.2514/6.2016-3935.

13. Ziniu, W., X. Yizhe, W. Wenbin, and H. Ruifeng. Review of shock wave detection method in CFD post-processing. Chinese Journal of Aeronautics, 2013, 26, 3, 501-13.

14. Kanamori, M., K. Suzuki. Shock Wave Detection based on the Theory of Characteristics for CFD Results. 20 $0^{\text {th }}$ AIAA Computational Fluid Dynamics Conference, 2011, DOI: https://doi.org/10.2514/6.2011-3681

15. Li, C., S. Zhu, Y. Xu, and Y. Xiao. 2.5D large eddy simulation of vertical axis wind turbine in consideration of high angle of attack flow, Renewable Energy, 2012, 51, 317-30.

16. Zhang, Y., H. Chen, S. Fu, and W. Dong. Numerical Study of an airfoil with riblets installed based on large eddy simulation. Aerospace Science and Technology, 2018, 78, 661-670.

17. Oro, J. M. F., A. Meana-Fernández, M. G. Vega, B. Pereiras, and J. G. Pérez. LES-based simulation of the time-resolved flow for rotor-stator interactions in axial fan stages. International Journal of Numerical Methods for Heat \& Fluid Flow, 2019, 29, $657-681$.

18. Rumsey, C. L., T. Nishino. Numerical study comparing RANS and LES approaches on a circulation control airfoil. International Journal of Heat \& Fluid Flow, 2011, 32, 5, 847-864.

19. Szydlowski, J., M. Costes. Simulation of Flow Around a Static and Oscillating in Pitch NACA 0015 Airfoil Using URANS and DES. ASME 2004 Heat Transfer/ Fluids Engineering Summer Conference, 2004, 2, 891-908. DOI: https://doi.org/10.1115/ HT-FED2004-56437.

20. Sagaut, P. Large Eddy Simulation for Incompressible Flows ( ${ }^{\text {rd }}$ Edition). Berlin, Springer-Verlag, 2006, 575 p. 